\title{
Efficacy of glutathione for the treatment of nonalcoholic fatty liver disease: an open-label, single-arm, multicenter, pilot study
}

Yasushi Honda ${ }^{1 \dagger}$, Takaomi Kessoku ${ }^{1 \dagger}$, Yoshio Sumida ${ }^{2}$, Takashi Kobayashi', Takayuki Kato ${ }^{1}$, Yuji Ogawa ${ }^{1}$, Wataru Tomeno ${ }^{1}$, Kento Imajo ${ }^{1}$, Koji Fujita', Masato Yoneda', Koshi Kataoka ${ }^{3}$, Masataka Taguri ${ }^{3}$, Takeharu Yamanaka ${ }^{3}$, Yuya Seko ${ }^{4}$, Saiyu Tanaka ${ }^{5}$, Satoru Saito ${ }^{1}$, Masafumi Ono ${ }^{6}$, Satoshi Oeda ${ }^{7}$, Yuichiro Eguchi ${ }^{7}$, Wataru Aoi ${ }^{8}$, Kenji Sato ${ }^{9}$, Yoshito Itoh $^{4}$ and Atsushi Nakajima ${ }^{1 *}$

\begin{abstract}
Background: Glutathione plays crucial roles in the detoxification and antioxidant systems of cells and has been used to treat acute poisoning and chronic liver diseases by intravenous injection. This is a first study examining the therapeutic effects of oral administration of glutathione in patients with nonalcoholic fatty liver disease (NAFLD).

Methods: The study was an open label, single arm, multicenter, pilot trial. Thirty-four NAFLD patients diagnosed using ultrasonography were prospectively evaluated. All patients first underwent intervention to improve their lifestyle habits (diet and exercise) for 3 months, followed by treatment with glutathione (300 mg/day) for 4 months. We evaluated their clinical parameters before and after glutathione treatment. We also quantified liver fat and fibrosis using vibrationcontrolled transient elastography. The primary outcome of the study was the change in alanine aminotransferase (ALT) levels.
\end{abstract}

Results: Twenty-nine patients finished the protocol. ALT levels significantly decreased following treatment with glutathione for 4 months. In addition, triglycerides, non-esterified fatty acids, and ferritin levels also decreased with glutathione treatment. Following dichotomization of ALT responders based on a median 12.9\% decrease from baseline, we found that ALT responders were younger in age and did not have severe diabetes compared with ALT non-responders. The controlled attenuation parameter also decreased in ALT responders.

Conclusions: This pilot study demonstrates the potential therapeutic effects of oral administration of glutathione in practical dose for patients with NAFLD. Large-scale clinical trials are needed to verify its efficacy.

Trial registration: UMIN000011118 (date of registration: July 4, 2013).

Keywords: Nonalcoholic fatty liver disease, Glutathione, Controlled attenuation parameter

\footnotetext{
* Correspondence: nakajima-tky@umin.ac.jp

${ }^{\dagger}$ Equal contributors

'Department of Gastroenterology and Hepatology, Yokohama City University

Graduate School of Medicine, Yokohama, Japan

Full list of author information is available at the end of the article
}

(c) The Author(s). 2017 Open Access This article is distributed under the terms of the Creative Commons Attribution 4.0 International License (http://creativecommons.org/licenses/by/4.0/), which permits unrestricted use, distribution, and reproduction in any medium, provided you give appropriate credit to the original author(s) and the source, provide a link to the Creative Commons license, and indicate if changes were made. The Creative Commons Public Domain Dedication waiver (http://creativecommons.org/publicdomain/zero/1.0/) applies to the data made available in this article, unless otherwise stated. 


\section{Background}

Nonalcoholic fatty liver disease (NAFLD) is an important cause of chronic liver injury worldwide $[1,2]$. The spectrum of NAFLD ranges from nonalcoholic fatty liver to nonalcoholic steatohepatitis (NASH), cirrhosis, and hepatocellular carcinoma [3]. NAFLD is associated with metabolic syndromes and the incidence of NAFLD has increased over time [4, 5]. Firstline treatment for NAFLD is lifestyle modification to achieve weight reduction, particularly through diet and exercise [6]. However, weight reduction is very difficult to accomplish and maintain. Effective therapy for NAFLD has not yet been established.

Glutathione, $\gamma$-L-glutamyl-L-cysteinyl-glycine, is a tripeptide present in every cell of the human body [7]. Although its functions are complex and remain the subject of current research, glutathione is thought to play crucial roles in the detoxification and antioxidant systems in cells. Because a reduction of glutathione levels in cells has been found to increase the risks for diseases and poisoning, direct intravenous injection of glutathione has been used to treat patients with chronic liver diseases and poisoning $[8,9]$.

Glutathione is synthesized in cells from glutamic acid, cysteine, and glycine. Cysteine and glycine are generated from methionine and serine, respectively, and glutamic acid is synthesized from $\alpha$-ketoglutarate, a metabolite of glucose. These amino acids are generally supplied from food. It has been reported that oral administration of glutathione did not change the levels of glutathione and glutathione disulfide in the deproteinized fraction of blood [10], and it has been suggested that orally administered glutathione is degraded into constituting amino acids and does not exert specific activity beyond the amino acid source. However, it has been reported that glutathione can pass through the mono layer of Caco-2 cells without degradation [11]. In addition, Park et al. reported an increase in the protein-bound form of glutathione in human blood after oral administration, while glutathione in the deproteinized fraction did not change [12]. These studies suggest that orally administered glutathione is absorbed into the blood and might have effects on the redox status in the human body. Such findings have encouraged us to examine the therapeutic effects of oral administration of glutathione on NAFLD.

The objective of the current study was to demonstrate the therapeutic potential of oral administration of glutathione in an open-label, single-arm, multicenter, pilot study prior to subsequent large-scale clinical trials. In this study, we compared clinical parameters before and after treatment with glutathione. We also evaluated controlled attenuation parameter (CAP) and liver stiffness measurement (LSM), as determined by vibration-controlled transient elastography (VCTE).

\section{Methods}

\section{Patients and study design}

The study protocol was conducted in accordance with the guidelines contained within the Declaration of Helsinki and was approved by the ethics committees of Yokohama City University and Kyoto Prefectural University of Medicine. Written informed consent was obtained from all participants before entry into the study. The trial is registered with the University Hospital Medical Information Network (UMIN) Clinical Trials Registry (UMIN000011118).

Patient enrollment began in January 2014 and ended when the target sample size was reached in September 2014. Follow-up of participants ended in December 2014. We prospectively evaluated 34 NAFLD patients with liver dysfunction. NAFLD was diagnosed based on ultrasonography. All 34 patients provided a detailed medical history and underwent a physical examination. Patients were excluded if they had infectious hepatitis (hepatitis B or C or Epstein-Barr virus infection), autoimmune hepatitis, primary biliary cirrhosis, sclerosing cholangitis, hemochromatosis, $\alpha 1$-antitrypsin deficiency, Wilson's disease, drug-induced hepatitis, alcoholic hepatitis, or excessive alcohol consumption (present or past consumption of $>20 \mathrm{~g}$ alcohol/day). No NAFLD patient had clinical evidence of hepatic decompensation, such as hepatic encephalopathy, ascites, variceal bleeding, or a serum bilirubin level greater than twice the upper limit of normal. All patients were started on a standard diet $(30 \mathrm{kcal} / \mathrm{kg} /$ day, consisting of $50-60 \%$ carbohydrate, $20-30 \%$ fat, and $15-20 \%$ protein) and received exercise counseling beginning 3 months before glutathione treatment. Exercise consisted of 5-6 metabolic equivalents for $30 \mathrm{~min}$ daily. Patients taking medication for lifestyle-related comorbid diseases, such as hypertension, dyslipidemia, and diabetes, were included; however, no change in medication or dose was allowed.

Because serum alanine aminotransferase (ALT) levels have been reported to predict the histological course of NASH and because strict control of ALT is required to prevent the progression of NASH [13], the primary outcome of this study was a change in ALT levels.

\section{Anthropometric and laboratory evaluations}

Patient weight and height were measured using a calibrated scale after patients removed their shoes and any heavy clothing. Venous blood samples were obtained after patients had fasted overnight $(12 \mathrm{~h})$. Platelet counts and concentrations of fasting blood sugar (FBS), hemoglobin A1c (HbA1c), immunoreactive insulin (IRI), high-density lipoprotein (HDL) cholesterol, low-density lipoprotein (LDL) cholesterol, triglycerides, nonesterified fatty acids (NEFA), aspartate aminotransferase (AST), ALT, $\gamma$-glutamyl transpeptidase, ferritin, and type 
IV collagen 7 were measured using standard laboratory techniques before and after glutathione treatment. Patients with FBS $\geq 126 \mathrm{mg} / \mathrm{dL}$, HbA1c $\geq 6.5 \%$, and/or currently using antidiabetic medication were defined as having diabetes according to the criteria of the Japan Diabetes Society [14].

Glutathione in the deproteinized fraction and proteinbound fraction of plasma were determined using the method described by Park et al. [12]. Briefly, $100 \mu \mathrm{L}$ of plasma was mixed with three parts ethanol. The supernatant was used as the deproteinized fraction. The precipitate was extracted using $100 \mu \mathrm{L}$ of $5 \%$ trichloroacetic containing 2\% 2-mercaptoethanol. The supernatant was used as the protein-bound fraction. Glutathione in these fractions were alkalized and derivatized with 6-aminoquinolyl-N hydroxy succinimidyl carbamate as described previously. The derivatives were resolved and detected using liquid chromatography/electron spray ionization/tandem mass spectrometry in multi-reaction monitoring mode.

\section{Vibration-controlled transient elastography}

VCTE was performed using an M-probe device (Fibroscan; EchoSens, Paris, France). Details of the technique and the examination procedure for LSM have been described previously $[15,16]$. CAP was measured using VCTE to stage steatosis. The technique is a proprietary algorithm based on the ultrasonic attenuation coefficient of the shear wave of VCTE, an estimate of the total ultrasonic attenuation at $3.5 \mathrm{MHz}$. CAP uses the same radiofrequency data as LSM and is only appraised if the acquisition is valid. It is expressed in decibels per meter. Measurements were obtained from the right lobe of the liver through the intercostal spaces, with a patient lying in the dorsal decubitus position and the right arm in maximal abduction. Only VCTE measurements based on at least 10 valid shots and success rates $\geq 60 \%$ were considered reliable and were used for statistical analysis.

\section{Statistical analysis}

Data are expressed as mean \pm standard deviation, unless indicated otherwise. The sample size was determined by reference to a previous report [17]. We estimated that with this sample size, the study would have $80 \%$ power to detect an absolute difference in the rate of improvement in ALT of 30 percentage points, with a two-sided type 1 error of 0.05 . All statistical analyses were performed using JMP ver. 11.2.0 software (SAS Institute, Cary, NC, USA). Univariate comparisons between patient groups were analyzed using the Student's $t$-test or the Mann-Whitney's Utest, as appropriate. A $p$-value $<0.05$ was considered statistically significant.

\section{Results}

\section{Biochemical response after 4 months of glutathione treatment}

The study flowchart is shown in Fig. 1. Of the 34 patients enrolled, two withdrew before the start of treatment. The remaining 32 were treated with L-glutathione (300 mg/day; KOHJIN Life Sciences, Tokyo, Japan, US FDA GRAS \#GRN000293) for 4 months by oral administration. Twenty-nine patients (14 men, 15 women, mean age $56.0 \pm 13.3$ years) finished the study protocol.

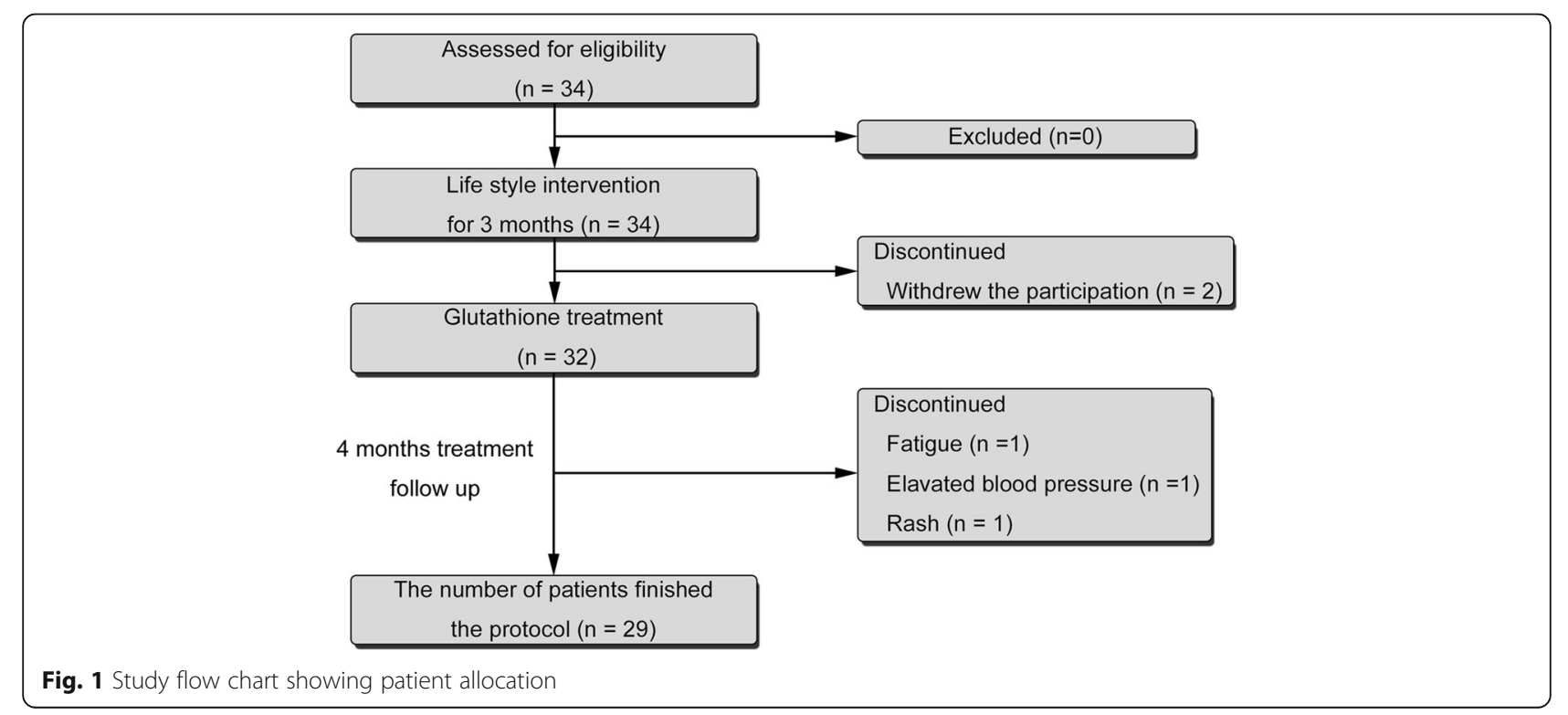


Table 1 Characteristics of patients before and after glutathione treatment $(n=29)$

\begin{tabular}{|c|c|c|c|}
\hline & Before treatment & After treatment & $P$-value \\
\hline Age (year) & $56.0 \pm 13.3$ & & - \\
\hline Male/female (n) & $14 / 15$ & & - \\
\hline Dyslipidemia (\%) & $24(82.8)$ & & - \\
\hline Statin (\%) & $12(41.4)$ & & - \\
\hline Diabetes (\%) & $14(48.3)$ & & - \\
\hline $\mathrm{BMI}\left(\mathrm{kg} / \mathrm{m}^{2}\right)$ & $26.5 \pm 3.9$ & $26.5 \pm 3.9$ & 0.32 \\
\hline FBS (mg/dL) & $118.4 \pm 34.3$ & $120.0 \pm 27.8$ & 0.24 \\
\hline IRI $(\mu \mathrm{U} / \mathrm{mL})$ & $23.1 \pm 29.8$ & $23.4 \pm 33.8$ & 0.38 \\
\hline $\mathrm{HbA}_{1 \mathrm{c}}(\%)$ & $6.37 \pm 1.18$ & $6.46 \pm 1.23$ & 0.016 \\
\hline HDL cholesterol (mg/dL) & $55.2 \pm 16.3$ & $55.0 \pm 15.4$ & 0.32 \\
\hline LDL cholesterol (mg/dL) & $114.0 \pm 28.8$ & $111.3 \pm 28.0$ & 0.08 \\
\hline Triglycerides (mg/dL) & $195.2 \pm 135.9$ & $163.6 \pm 121.9$ & 0.007 \\
\hline NEFA ( $\mu \mathrm{Eq} / \mathrm{L})$ & $651.2 \pm 242.5$ & $533.5 \pm 209.7$ & 0.013 \\
\hline AST (IU/L) & $46.7 \pm 17.2$ & $47.6 \pm 21.2$ & 0.39 \\
\hline ALT (IU/L) & $68.9 \pm 36.1$ & $58.1 \pm 33.5$ & 0.014 \\
\hline GGT (IU/L) & $70.4 \pm 46.5$ & $66.6 \pm 47.5$ & 0.29 \\
\hline Ferritin (ng/mL) & $219.8 \pm 150.8$ & $194.4 \pm 139.2$ & 0.015 \\
\hline Platelet count $\left(\times 10^{4} / \mu \mathrm{L}\right)$ & $20.8 \pm 5.7$ & $20.9 \pm 5.3$ & 0.30 \\
\hline Type IV collagen 7 s & $5.08 \pm 1.95$ & $4.84 \pm 1.34$ & 0.40 \\
\hline Glutathione in protein fraction $(\mu \mathrm{M})$ & $1.42 \pm 0.87$ & $0.93 \pm 0.63$ & 0.010 \\
\hline Glutathione in deproteinized fraction $(\mu \mathrm{M})$ & $0.025 \pm 0.040$ & $0.019 \pm 0.024$ & 0.27 \\
\hline CAP $(\mathrm{db} / \mathrm{m})$ & $295.7 \pm 44.9$ & $285.4 \pm 48.8$ & 0.07 \\
\hline LSM (kPa) & $9.94 \pm 4.93$ & $9.24 \pm 4.48$ & 0.16 \\
\hline
\end{tabular}

Data are expressed as mean \pm standard deviation. BMI body mass index, FBS fasting blood sugar, IRI immunoreactive insulin, $H B A 1 C$, hemoglobin A1C, HDL cholesterol high-density lipoprotein cholesterol, $L D L$ cholesterol low-density lipoprotein cholesterol, NEFA non-esterified fatty acid, AST aspartate aminotransferase, $A L T$ alanine aminotransferase, GGT $\mathrm{Y}$-glutamyl transpeptidase, CAP controlled attenuation parameter, LSM liver stiffness measurement

Three patients dropped out, one each owing to fatigue, elevated blood pressure, and rash.

The clinical and laboratory characteristics of the study participants are shown in Table 1. Twenty-four patients (82.8\%) had dyslipidemia and $12(41.4 \%)$ were taking statins. Fourteen patients (48.3\%) had diabetes.

After 4 months of glutathione treatment, ALT levels decreased significantly. Glutathione treatment decreased the concentrations of triglycerides, NEFA, and ferritin. HbA1c levels increased after glutathione treatment. Unexpectedly, glutathione in the plasma protein fraction decreased significantly after glutathione treatment. There was no significant difference in glutathione levels in the deproteinized fraction. Although glutathione treatment did not significantly affect CAP and LSM values, both tended to decrease.

\section{Glutathione treatment improved CAP values in ALT responders}

The median decrease in ALT level from baseline was $12.9 \%$. The 29 patients were divided into ALT responders $(n=15)$, defined as those with an ALT reduction $\geq 12.9 \%$, and ALT non-responders $(n=14)$, defined as those with an ALT reduction $<12.9 \%$, and the factors associated with responses to glutathione were evaluated (Table 2). ALT responders were significantly younger than ALT non-responders $(50.7 \pm 12.1$ years vs. $61.7 \pm 12.4$ years, $p=0.011$ ). Body mass index (BMI) did not differ between ALT responders and non-responders $\left(26.5 \pm 4.1 \mathrm{~kg} / \mathrm{m}^{2}\right.$ vs. $\left.26.6 \pm 3.8 \mathrm{~kg} / \mathrm{m}^{2}, p=0.47\right)$. Although the percentages of ALT responders and non-responders with dyslipidemia did not differ $(80.0 \%$ vs. $85.7 \%$, $p=0.68)$, HDL cholesterol and LDL cholesterol levels were higher in ALT responders. Rates of statin use tended to be lower in ALT responders than in non-responders $(26.7 \%$ vs. $57.1 \%, p=0.10)$. Rates of diabetes also tended to be lower $(33.3 \%$ vs. $64.3 \%, p=0.10)$ and HbA1c levels were significantly lower in ALT responders compared with non-responders. There were no significant differences in glutathione levels in the plasma protein and deproteinized fractions between ALT responders and non-responders before glutathione treatment.

The characteristics of ALT responders and nonresponders before and after glutathione treatment are 
Table 2 Characteristics of ALT responders and non-responders

\begin{tabular}{|c|c|c|c|}
\hline & ALT responders $(n=15)$ & ALT non-responders $(n=14)$ & $P$-value \\
\hline Age (year) & $50.7 \pm 12.1$ & $61.7 \pm 12.4$ & 0.011 \\
\hline Male/female (n) & $9(6)$ & $5(9)$ & 0.191 \\
\hline Dyslipidemia (\%) & $12(80.0)$ & $12(85.7)$ & 0.68 \\
\hline Statin (\%) & $4(26.7)$ & $8(57.1)$ & 0.10 \\
\hline Diabetes (\%) & $5(33.3)$ & $9(64.3)$ & 0.10 \\
\hline BMI $\left(\mathrm{kg} / \mathrm{m}^{2}\right)$ & $26.5 \pm 4.1$ & $26.6 \pm 3.8$ & 0.47 \\
\hline $\mathrm{FBS}(\mathrm{mg} / \mathrm{dL})$ & $115.7 \pm 37.9$ & $121.4 \pm 31.2$ & 0.33 \\
\hline IRI $(\mu \mathrm{U} / \mathrm{mL})$ & $23.5 \pm 22.7$ & $22.6 \pm 38.0$ & 0.47 \\
\hline $\mathrm{HbA}_{1 \mathrm{c}}(\%)$ & $5.94 \pm 1.03$ & $6.9 \pm 1.2$ & 0.019 \\
\hline HDL cholesterol (mg/dL) & $60.3 \pm 18.6$ & $49.7 \pm 11.7$ & 0.04 \\
\hline LDL cholesterol (mg/dL) & $124.5 \pm 33.1$ & $102.9 \pm 18.6$ & 0.021 \\
\hline Triglycerides (mg/dL) & $202.4 \pm 164.1$ & $187.6 \pm 103.3$ & 0.39 \\
\hline NEFA $(\mu \mathrm{Eq} / \mathrm{L})$ & $720.2 \pm 285.0$ & $563.3 \pm 143.0$ & 0.055 \\
\hline AST (IU/L) & $46.3 \pm 20.2$ & $47.1 \pm 14.1$ & 0.45 \\
\hline ALT (IU/L) & $77.1 \pm 38.6$ & $60.1 \pm 32.2$ & 0.104 \\
\hline GGT (IU/L) & $81.9 \pm 58.0$ & $58.0 \pm 26.6$ & 0.085 \\
\hline Ferritin (ng/mL) & $260.2 \pm 164.5$ & $176.6 \pm 126.1$ & 0.07 \\
\hline Platelet count $\left(\times 10^{4} / \mu \mathrm{L}\right)$ & $20.3 \pm 4.6$ & $21.3 \pm 6.8$ & 0.32 \\
\hline Type IV collagen $7 \mathrm{~s}$ & $4.61 \pm 1.13$ & $5.59 \pm 2.51$ & 0.09 \\
\hline Glutathione in protein fraction $(\mu \mathrm{M})$ & $1.53 \pm 0.92$ & $1.27 \pm 0.83$ & 0.230 \\
\hline Glutathione in deprotenized fraction $(\mu \mathrm{M})$ & $0.017 \pm 0.020$ & $0.036 \pm 0.057$ & 0.116 \\
\hline CAP $(\mathrm{db} / \mathrm{m})$ & $300.3 \pm 41.1$ & $290.4 \pm 50.2$ & 0.29 \\
\hline LSM (kPa) & $8.71 \pm 4.63$ & $11.36 \pm 5.05$ & 0.080 \\
\hline
\end{tabular}

Data are expressed as mean \pm standard deviation

Abbreviations: $B M I$ body mass index, FBS fasting blood sugar, IRI immunoreactive insulin, $H B A 1 \mathrm{c}$ hemoglobin $A 1 \mathrm{c}, H D L$ cholesterol high density lipoprotein cholesterol, $L D L$ cholesterol low density lipoprotein cholesterol, NEFA non-esterified fatty acid, AST asparate aminotransferase, ALT alanine aminotransferase, GGT $Y$-glutamyl transpeptidase, CAP controlled attenuation parameter, LSM liver stiffness measurement

shown in Table 3. Glutathione treatment decreased ALT levels in ALT responders (Fig. 2a) but increased AST and ALT levels in ALT non-responders (Fig. 2b). In ALT responders, glutathione treatment decreased NEFA, ferritin, and HDL cholesterol levels but increased HbA1c levels. In ALT non-responders, glutathione treatment reduced triglyceride levels but increased FBS levels. Glutathione treatment significantly decreased glutathione in the plasma protein-boud fraction in ALT responders; there was no change in ALT non-responders. Surprisingly, CAP values were significantly reduced in ALT responders; there were no differences in ALT non-responders.

\section{Discussion}

Glutathione has a long history for the treatment of chronic liver disease by intravenous injection. This study demonstrates a therapeutic effect of glutathione by oral administration in patients with NAFLD. The primary outcome of this study was a change in ALT levels. The 29 patients who were treated with oral administration of glutathione $(300 \mathrm{mg} /$ day $)$ for 4 months showed a reduction in ALT levels as well as reductions in triglycerides, NEFA, and ferritin levels. The findings of the current study suggest the beneficial effects of glutathione by oral administration for NAFLD patients. It is thought that glutathione is degraded into amino acids during digestion and absorption processes. Orally administered glutathione is suggested to serve as a source of amino acids in the synthesis of endogenous glutathione. Supplementation of large doses of glycine and serine, precursors of glutathione, can attenuate NAFLD in human and animal models $[18,19]$. In the current study, the dose of glutathione was $300 \mathrm{mg} /$ day. The amount of cysteine potentially released from $300 \mathrm{mg}$ of glutathione is less than $120 \mathrm{mg}$, the amount that can be obtained from 10 to $20 \mathrm{~g}$ of meat or $100 \mathrm{~mL}$ of milk. It is, therefore, very unlikely that the current dose of orally administered glutathione attenuates the pathogenesis of NAFLD via an amino acid source for glutathione synthesis.

It is reported that the level of the protein-bound form of glutathione increases 1-2 h after ingestion of glutathione, which suggests that orally administered glutathione is 
Table 3 Characteristics of ALT responders and non-responders before and after glutathione treatment

\begin{tabular}{|c|c|c|c|c|c|c|}
\hline & ALT responders $(n$ & 15) & & ALT non-responde & $(n=14)$ & \\
\hline & Before treatment & After treatment & $P$-value & Before treatment & After treatment & $P$-value \\
\hline Age (year) & $50.7 \pm 12.1$ & & - & $61.7 \pm 12.4$ & & - \\
\hline Male/female (n) & $9(6)$ & & - & $5(9)$ & & - \\
\hline Dyslipidemia (\%) & $12(80.0)$ & & - & $12(85.7)$ & & - \\
\hline Statin (\%) & $4(26.7)$ & & - & $8(57.1)$ & & - \\
\hline Diabetes (\%) & $5(33.3)$ & & - & $9(64.3)$ & & - \\
\hline $\mathrm{BMI}\left(\mathrm{kg} / \mathrm{m}^{2}\right)$ & $26.5 \pm 4.1$ & $26.5 \pm 4.0$ & 0.23 & $26.6 \pm 3.8$ & $26.4 \pm 3.9$ & 0.45 \\
\hline $\mathrm{FBS}(\mathrm{mg} / \mathrm{dL})$ & $115.7 \pm 37.9$ & $113.0 \pm 23.0$ & 0.38 & $121.4 \pm 31.2$ & $128.2 \pm 31.4$ & 0.004 \\
\hline $\mid \mathrm{RI}(\mu \mathrm{U} / \mathrm{mL})$ & $23.5 \pm 22.7$ & $17.9 \pm 14.3$ & 0.14 & $22.6 \pm 38.0$ & $30.5 \pm 31.4$ & 0.11 \\
\hline $\mathrm{HbA}_{1 c}(\%)$ & $5.94 \pm 1.03$ & $6.08 \pm 1.10$ & 0.017 & $6.9 \pm 1.2$ & $6.89 \pm 1.28$ & 0.14 \\
\hline HDL cholesterol (mg/dL) & $60.3 \pm 18.6$ & $57.4 \pm 16.7$ & 0.001 & $49.7 \pm 11.7$ & $52.2 \pm 13.8$ & 0.08 \\
\hline LDL cholesterol (mg/dL) & $124.5 \pm 33.1$ & $117.1 \pm 34.8$ & 0.06 & $102.9 \pm 18.6$ & $104.1 \pm 14.5$ & 0.41 \\
\hline Triglycerides (mg/dL) & $202.4 \pm 164.1$ & $178.8 \pm 157.6$ & 0.15 & $187.6 \pm 103.3$ & $146.2 \pm 62.1$ & 0.003 \\
\hline NEFA ( $\mu \mathrm{Eq} / \mathrm{L})$ & $720.2 \pm 285.0$ & $576.0 \pm 230.1$ & 0.032 & $563.3 \pm 143.0$ & $473.9 \pm 170.7$ & 0.13 \\
\hline AST (IU/L) & $46.3 \pm 20.2$ & $40.0 \pm 20.7$ & 0.11 & $47.1 \pm 14.1$ & $55.8 \pm 19.3$ & 0.003 \\
\hline $\mathrm{ALT}(\mathrm{IU} / \mathrm{L})$ & $77.1 \pm 38.6$ & $47.9 \pm 28.1$ & $<0.0001$ & $60.1 \pm 32.2$ & $68.9 \pm 36.3$ & 0.005 \\
\hline GGT (IU/L) & $81.9 \pm 58.0$ & $64.8 \pm 48.4$ & 0.07 & $58.0 \pm 26.6$ & $68.5 \pm 48.3$ & 0.06 \\
\hline Ferritin (ng/mL) & $260.2 \pm 164.5$ & $217.7 \pm 162.3$ & 0.015 & $176.6 \pm 126.1$ & $169.5 \pm 109.9$ & 0.28 \\
\hline Platelet count $\left(\times 10^{4} / \mu \mathrm{L}\right)$ & $20.3 \pm 4.6$ & $20.2 \pm 4.7$ & 0.45 & $21.3 \pm 6.8$ & $21.8 \pm 6.0$ & 0.25 \\
\hline Type IV collagen $7 \mathrm{~s}$ & $4.61 \pm 1.13$ & $4.39 \pm 1.06$ & 0.10 & $5.59 \pm 2.51$ & $5.42 \pm 1.49$ & 0.13 \\
\hline Glutathione in protein fraction $(\mu \mathrm{M})$ & $1.53 \pm 0.92$ & $0.88 \pm 0.53$ & 0.004 & $1.27 \pm 0.83$ & $1.00 \pm 0.77$ & 0.24 \\
\hline Glutathione in deprotenized fraction $(\mu \mathrm{M})$ & $0.017 \pm 0.020$ & $0.019 \pm 0.029$ & 0.60 & $0.036 \pm 0.057$ & $0.018 \pm 0.017$ & 0.19 \\
\hline $\operatorname{CAP}(\mathrm{db} / \mathrm{m})$ & $300.3 \pm 41.1$ & $285.1 \pm 53.2$ & 0.049 & $290.4 \pm 50.2$ & $285.8 \pm 44.9$ & 0.31 \\
\hline $\mathrm{LSM}(\mathrm{kPa})$ & $8.71 \pm 4.63$ & $7.91 \pm 4.22$ & 0.19 & $11.36 \pm 5.05$ & $10.9 \pm 4.38$ & 0.32 \\
\hline
\end{tabular}

Data are expressed as mean \pm standard deviation

Abbreviations: $B M I$ body mass index, $F B S$ fasting blood sugar, $I R I$ immunoreactive insulin, $H B A 1 c$ hemoglobin $A 1 c$, $H D L$ cholesterol high density lipoprotein cholesterol, $L D L$ cholesterol low density lipoprotein cholesterol, NEFA non-esterified fatty acid, AST aspartate aminotransferase, ALT alanine aminotransferase, GGT $\gamma$-glutamyl transpeptidase, CAP controlled attenuation parameter, LSM liver stiffness measurement

absorbed into the blood [12]. This protein-bound glutathione may be deposited in the liver, attenuating hepatitis.

The levels of protein-bound glutathione were reported to return to baseline levels after an overnight fast [12]. In the current study, we found that the baseline level of the protein-bound form of glutathione significantly decreased after an overnight fast following 4 months of glutathione administration, especially in ALT responders. The levels of protein-bound glutathione in patients in the current study were considerably higher than those of healthy
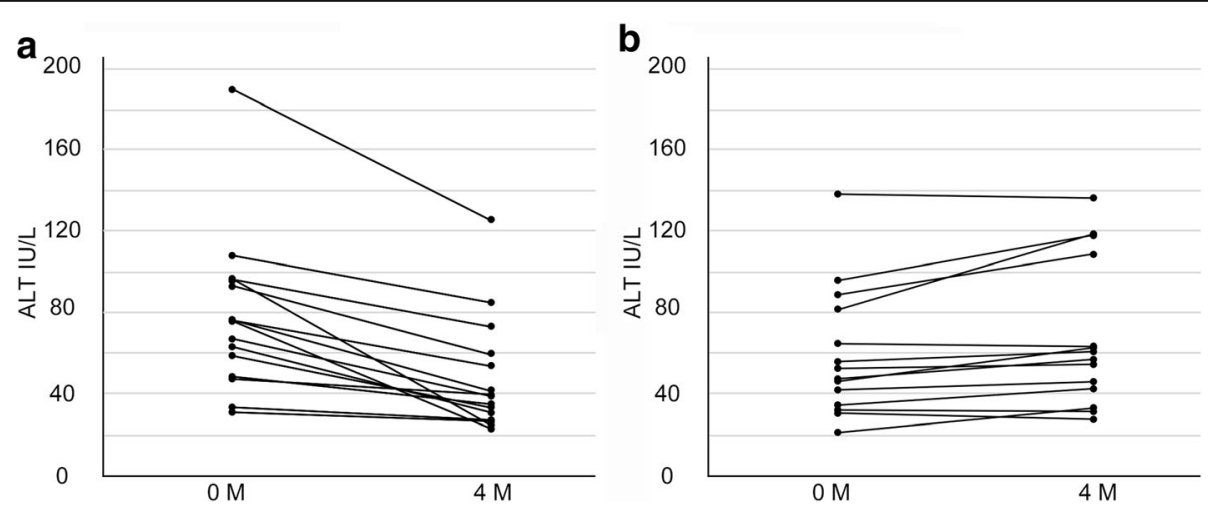

Fig. 2 Alanine aminotransferase (ALT) levels before and after treatment with glutathione in a ALT responders and $\mathbf{b}$ ALT non-responders 
volunteers in previous studies [12] estimated using the same method. Glutathione treatment also decreased protein-bound glutathione to normal baseline levels. These findings suggest that oral administration of glutathione may increase the incorporation of protein-bound glutathione into the liver or decrease the pathological excretion of glutathione from the liver.

NAFLD is a complex disease. Its pathogenesis is thought to involve various factors, including insulin resistance, lipotoxicity, gut/nutrient-derived signals, adipocytokines, oxidative stress, and genetic factors. Dyslipidemia has been reported in $20-80 \%$ of patients with NAFLD [20]. Our previously study revealed that orally administrated glutathione accelerates fatty acid utilization by upregulating levels of the protein peroxisome proliferator-activated receptor- $\gamma$ coactivator- $1 \alpha$ and mitochondrial DNA with reduced plasma NEFA levels [21]. The current study also revealed that $24(82.8 \%)$ of our patients had dyslipidemia, and glutathione treatment reduced triglyceride and NEFA levels significantly.

Increases in ferritin and body iron stores have been detected frequently in NAFLD patients [22, 23]. Ferritin and iron can promote the development of NAFLD through oxidative stress [24]. Results from the PIVENS trial showed that oral administration of the anti-oxidant vitamin $\mathrm{E}$ improved liver dysfunction and the pathological conditions of NASH [17]. However, long-term treatment with vitamin $\mathrm{E}$ has been associated with increases in all-cause mortality and the risk for prostate cancer [25-27], suggesting the need to evaluate the efficacy and safety of this agent. In the current study, glutathione treatment significantly decreased ferritin levels, but the mechanism behind the decrease remains unclear. Glutathione is thought to ameliorate hyperferritinemia and oxidative stress, and to have therapeutic effects in patients with NAFLD.

Liver fat was non-invasively assessed using VCTE with CAP. A meta-analysis found that CAP has good sensitivity and specificity for detecting liver fat [28]. CAP values in our study tended to decrease in all patients and significantly decreased in ALT responders following 4 months of glutathione treatment. Although the relationship between histologic improvement of hepatic steatosis and the reduction of CAP values has not yet been determined, glutathione may reduce hepatic steatosis.

We also investigated the patient factors associated with the therapeutic effects of glutathione. We found that HDL cholesterol and LDL cholesterol levels were higher and HbA1c levels lower in ALT responders than in non-responders. Although the percentage of patients using statins did not differ significantly between the two groups, the percentage tended to be lower in ALT responders than in ALT non-responders. While it can be nothing more than speculation because of the small sample size, patients who showed therapeutic effects following glutathione treatment appeared to be younger and did not have diabetes or had mild diabetes.

Three patients withdrew from the study because of fatigue, elevated blood pressure, and a rash. In ALT responders, HbA1c levels increased and HDL cholesterol levels decreased after glutathione treatment. A study of 6522 patients found that $24(0.4 \%)$ had experienced adverse reactions, the most frequent being anorexia, nausea, vomiting, and rash [29]. Although administration of glutathione may have been associated with a rash in one patient in the current study, the causal associations between glutathione and other adverse effects are unclear.

This study had some limitations. First, our study was a single-arm study without a control group. Second, the study was limited by the small sample size and the short treatment period (4 months). Third, as the pathological conditions of the patients were not evaluated by liver biopsy, incorporation of orally administered glutathione in the liver was not confirmed. Fourth, a number of patients withdrew from the study but no causal association can be determined.

\section{Conclusions}

Treatment with glutathione significantly improved ALT levels. In addition, CAP values were significantly reduced in ALT responders. Our pilot study suggests that oral administration of glutathione supports hepatic metabolism and improves NAFLD. To elucidate the mechanism behind the beneficial effects of glutathione, further studies that examine the incorporation of orally administrated glutathione into the liver and the effects on the host redox system using stable isotope-labeled glutathione and animal models are required. Large-scale clinical trials are necessary to confirm the therapeutic effects of glutathione.

\begin{abstract}
Abbreviations
ALT: Alanine aminotransferase; AST: Aspartate aminotransferase; BMI: Body mass index; CAP: Controlled attenuation parameter; FBS: Fasting blood sugar; HbA1c: hemoglobin A1c; HDL: High-density lipoprotein; IRI: Immunoreactive insulin; LDL: Low-density lipoprotein; LSM: Liver stiffness measurement; NAFLD: Nonalcoholic fatty liver disease; NASH: Nonalcoholic steatohepatitis; NEFA: Non-esterified fatty acid; UMIN: University Hospital Medical Information Network; VCTE: Vibration-controlled transient elastography
\end{abstract}

\section{Acknowledgments}

This work was supported in part by the Japan Study Group of NAFLD (JSG-NAFLD, Kyoto, Japan).

\section{Funding}

KOHJIN Life Sciences (Tokyo, Japan). a subsidiary of Mitsubishi Corp. Life Sciences, provided glutathione and partial financial support for this work. KOHJIN Life Sciences was not involved in data analysis or manuscript preparation. 


\section{Availability of data and materials}

The data generated and analyzed during this study are available from the corresponding author on reasonable request.

\section{Authors' contributions}

Designed and coordinated the study: TK, YH, YS, and AN. Performed the experiments: TK, YH, YS, TK, TK, YO, WT, KI, KF, MY, YS, ST, SS, MO, SO, YE, YI, WA, KS, and AN. Performed the statistical analyses: TK, YH, and AN. Collected the data and critically reviewed the manuscript: TK, YH, YS, KK, MT, TY, WA, $\mathrm{KS}$, and AN. Wrote the manuscript: TK, YH, YS, WA, KS, and AN. All authors approved the final version of the manuscript.

\section{Ethics approval and consent to participate}

The study protocol was conducted according to the guidelines contained within the Declaration of Helsinki and was approved by the ethics committees of Yokohama City University and Kyoto Prefectural University. Written informed consent was obtained from all participants before entry into the study.

\section{Consent for publication}

Not applicable.

\section{Competing interests}

The authors declare that they have no competing interests.

\section{Publisher's Note}

Springer Nature remains neutral with regard to jurisdictional claims in published maps and institutional affiliations.

\section{Author details}

'Department of Gastroenterology and Hepatology, Yokohama City University Graduate School of Medicine, Yokohama, Japan. 'Division of Hepatology and Pancreatology, Department of Internal Medicine, Aichi Medical University, Aichi, Japan. ${ }^{3}$ Department of Biostatistics, Yokohama City University Graduate School of Medicine, Yokohama, Japan. ${ }^{4}$ Department of Gastroenterology and Hepatology, Kyoto Prefectural University of Medicine, Kyoto, Japan. ${ }^{5}$ Center for Digestive and Liver Diseases, Nara City Hospital, Nara, Japan. ${ }^{6}$ Department of Gastroenterology and Hepatology, Kochi Medical School, Kochi, Japan. ${ }^{7}$ Liver Center, Saga University Hospital, Saga, Japan. ${ }^{8}$ Division of Applied Life Sciences, Graduate School of Life and Environmental Sciences, Kyoto Prefectural University, Kyoto, Japan. ${ }^{9}$ Division of Applied Biosciences, Graduate School of Agriculture, Kyoto University, Kyoto, Japan.

Received: 1 March 2017 Accepted: 31 July 2017

Published online: 08 August 2017

\section{References}

1. Day CP. Non-alcoholic steatohepatitis (NASH): where are we now and where are we going? Gut. 2002;50:585-8.

2. Lazo M, Clark JM. The epidemiology of nonalcoholic fatty liver disease: a global perspective. Semin Liver Dis. 2008:28:339-50.

3. Neuschwander-Tetri BA, Caldwell SH. Nonalcoholic steatohepatitis: summary of an AASLD single topic conference. Hepatology. 2003;37:1202-19.

4. Marchesini $\mathrm{G}$, Bugianesi $\mathrm{E}$, Forlani $\mathrm{G}$, et al. Nonalcoholic fatty liver, steatohepatitis, and the metabolic syndrome. Hepatology. 2003;37:917-23.

5. Yilmaz Y, Younossi ZM. Obesity-associated nonalcoholic fatty liver disease. Clin Liver Dis. 2014;18:19-31.

6. Promrat $\mathrm{K}$, Kleiner $\mathrm{DE}$, Niemeier $\mathrm{HM}$, et al. Randomized controlled trial testing the effects of weight loss on nonalcoholic steatohepatitis. Hepatology. 2010;51:121-9.

7. Anderson ME. Glutathione: an overview of biosynthesis and modulation. Chem Biol Interact. 1998;111-112:1-14.

8. Dentico P, Volpe A, Buongiorno R, et al. Glutathione in the treatment of chronic fatty liver diseases. Recenti Prog Med. 1995;86:290-3. [in Italian]

9. Altomare E, Colonna P, D'Agostino C, et al. High-dose antioxidant therapy during thrombolysis in patients with acute myocardial infarction. Curr Ther Res Clin Exp. 1996;57:131-41.

10. Allen J, Bradley RD. Effects of oral glutathione supplementation on systemic oxidative stress biomarkers in human volunteers. J Altern Complement Med. 2011;17:827-33.

11. Kovacs-Nolan J, Rupa P, Matsui T, et al. In vitro and ex vivo uptake of glutathione (GSH) across the intestinal epithelium and fate of oral GSH after in vivo supplementation. J Agric Food Chem. 2014;62:9499-506.
12. Park EY, Shimura N, Konishi T, et al. Increase in the protein-bound form of glutathione in human blood after the oral administration of glutathione. J Agric Food Chem. 2014;62:6183-9.

13. Seko Y, Sumida Y, Tanaka S, et al. Serum alanine aminotransferase predicts the histological course of non-alcoholic steatohepatitis in Japanese patients. Hepatol Res. 2015;45:E53-61.

14. Committee of the Japan Diabetes Society on the Diagnostic Criteria of Diabetes Mellitus, Seino Y, Nanjo K, Tajima, et al. Report of the committee on the classification and diagnostic criteria of diabetes mellitus. J Diabetes Investig. 2010;1:212-28.

15. Sandrin L, Tanter M, Gennisson JL, et al. Shear elasticity probe for soft tissues with 1-D transient elastography. IEEE Trans Ultrason Ferroelectr Freq Control. 2002:49:436-46.

16. Sandrin L, Fourquet B, Hasquenoph JM, et al. Transient elastography: a new noninvasive method for assessment of hepatic fibrosis. Ultrasound Med Biol. 2003;29:1705-13.

17. Sanyal AJ, Chalasani N, Kowdley KV, et al. NASH CRN. Pioglitazone, vitamin E, or placebo for nonalcoholic steatohepatitis. N Engl J Med. 2010;362:1675-85.

18. Zhou $X$, Han $D, X u R$, et al. Glycine protects against high sucrose and high fat-induced non-alcoholic steatohepatitis in rats. Oncotarget. 2016;7:80223-37.

19. Mardinoglu A, Bjornson E, Zhang C, et al. Personal model-assisted identification of NAD+ and glutathione metabolism as intervention target in NAFLD. Mol Syst Biol. 2017;13:916.

20. Souza MR, Diniz Mde F, Medeiros-Filho JE, et al. Metabolic syndrome and risk factors for non-alcoholic fatty liver disease. Arq Gastroenterol. 2012:49:89-96.

21. Aoi W, Ogaya Y, Takami M, et al. Glutathione supplementation suppresses muscle fatigue induced by prolonged exercise via improved aerobic metabolism. J Int Soc Sports Nutr. 2015;12:7.

22. Valenti L, Fracanzani AL, Dongiovanni P, et al. Iron depletion by phlebotomy improves insulin resistance in patients with nonalcoholic fatty liver disease and hyperferritinemia: evidence from a case-control study. Am J Gastroenterol. 2007;102:1251-8

23. Lapenna D, Pierdomenico SD, Ciofani G, et al. Association of body iron stores with low molecular weight iron and oxidant damage of human atherosclerotic plaques. Free Radic Biol Med. 2007;42:492-8.

24. Dongiovanni P, Valenti L, Ludovica Fracanzani A, et al. Iron depletion by deferoxamine up-regulates glucose uptake and insulin signaling in hepatoma cells and in rat liver. Am J Pathol. 2008:172:738-47.

25. Miller ER 3rd, Pastor-Barriuso R, Dalal D, et al. Meta-analysis: high-dosage vitamin E supplementation may increase all-cause mortality. Ann Intern Med. 2005;142:37-46.

26. Bjelakovic G, Nikolova D, Gluud LL, et al. Mortality in randomized trials of antioxidant supplements for primary and secondary prevention: systematic review and meta-analysis. JAMA. 2007;297:842-57.

27. Klein EA, Thompson IM Jr, Tangen CM, et al. Vitamin E and the risk of prostate cancer: the selenium and vitamin E cancer prevention trial (SELECT). JAMA. 2011:306:1549-56.

28. Shi KQ, Tang JZ, Zhu XL, et al. Controlled attenuation parameter for the detection of steatosis severity in chronic liver disease: a meta-analysis of diagnostic accuracy. J Gastroenterol Hepatol. 2014;29:1149-58.

29. Pharmaceutical Interview Form, Japan Standard Commodity No. 873922, 2014.

\section{Submit your next manuscript to BioMed Central and we will help you at every step:}

- We accept pre-submission inquiries

- Our selector tool helps you to find the most relevant journal

- We provide round the clock customer support

- Convenient online submission

- Thorough peer review

- Inclusion in PubMed and all major indexing services

- Maximum visibility for your research

Submit your manuscript at www.biomedcentral.com/submit
) Biomed Central 